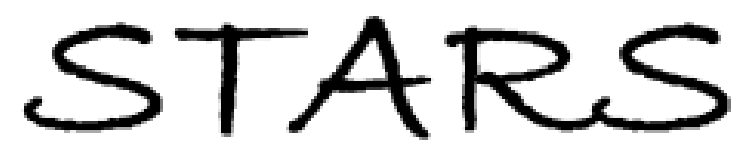

University of Central Florida

STARS

$1-1-2008$

\title{
Single cell gap and wide-view transflective liquid crystal display using fringe field switching and embedded wire grid polarizer
}

\author{
Zhibing Ge \\ University of Central Florida \\ Thomas X. Wu \\ University of Central Florida \\ Shin-Tson Wu \\ University of Central Florida
}

Find similar works at: https://stars.library.ucf.edu/facultybib2000

University of Central Florida Libraries http://library.ucf.edu

This Article is brought to you for free and open access by the Faculty Bibliography at STARS. It has been accepted for inclusion in Faculty Bibliography 2000 s by an authorized administrator of STARS. For more information, please contactSTARS@ucf.edu.

\section{Recommended Citation}

Ge, Zhibing; Wu, Thomas X.; and Wu, Shin-Tson, "Single cell gap and wide-view transflective liquid crystal display using fringe field switching and embedded wire grid polarizer" (2008). Faculty Bibliography 2000 s. 363.

https://stars.library.ucf.edu/facultybib2000/363

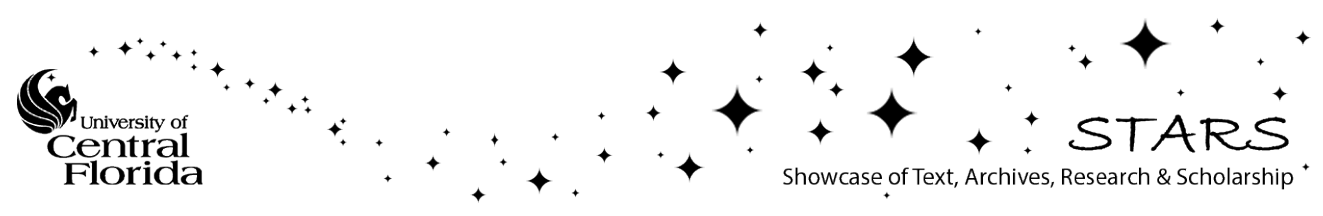




\section{Single cell gap and wide-view transflective liquid crystal display using fringe field switching and embedded wire grid polarizer}

Cite as: Appl. Phys. Lett. 92, 051109 (2008); https://doi.org/10.1063/1.2841847

Submitted: 06 December 2007 . Accepted: 18 January 2008 . Published Online: 06 February 2008

Zhibing Ge, Thomas X. Wu, and Shin-Tson Wu

\section{ARTICLES YOU MAY BE INTERESTED IN}

Nanowire grid polarizer for energy efficient and wide-view liquid crystal displays

Applied Physics Letters 93, 121104 (2008); https://doi.org/10.1063/1.2988267

Optical wire-grid polarizers at oblique angles of incidence

Journal of Applied Physics 93, 4407 (2003); https://doi.org/10.1063/1.1559937

30-nm-wide aluminum nanowire grid for ultrahigh contrast and transmittance polarizers made by UV-nanoimprint lithography

Applied Physics Letters 89, 141105 (2006); https://doi.org/10.1063/1.2358813

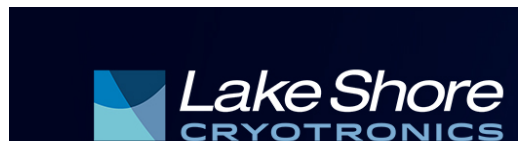

Sensors, Controllers, Monitors

from the world leader in cryogenic thermometry 


\title{
Single cell gap and wide-view transflective liquid crystal display using fringe field switching and embedded wire grid polarizer
}

\author{
Zhibing Ge, Thomas X. Wu, and Shin-Tson $\mathrm{Wu}^{\mathrm{a})}$ \\ College of Optics and Photonics, University of Central Florida, Orlando, Florida 32816, USA
}

(Received 6 December 2007; accepted 18 January 2008; published online 6 February 2008)

\begin{abstract}
A single cell gap and wide-view transflective liquid crystal display (TR-LCD) using fringe field switching and embedded wire grid polarizer as polarization dependent reflector is developed. Such a device structure does not require any quarter-wave plate or in-cell phase retarder. Through optimizing the pixel electrode width and gap, high transmittance and reflectance and a single gamma curve are obtained. Potential application of this TR-LCD for mobile displays is emphasized. (C) 2008 American Institute of Physics. [DOI: 10.1063/1.2841847]
\end{abstract}

Transflective liquid crystal displays (TR-LCDs) have been widely used for mobile displays because of their good sunlight readability and low power consumption. ${ }^{1}$ Most of TR-LCDs consist of transmissive $(T)$ and reflective $(R)$ modes. The $T$ mode uses backlight while $R$ mode uses ambient light to readout the displayed images. In the $T$ mode, the backlight passes the LC layer once while the ambient light traverses the LC layer twice. To compensate for this optical path length difference, both dual-cell-gap and singlecell-gap approaches have been developed. ${ }^{1}$ To achieve wideview, multiple-domain vertical alignment $(\mathrm{VA})^{2,3}$ and fringe field switching (FFS) (Ref. 4) have been explored. For a hand-held LCD, the demand in viewing angle is less stringent than a large screen LCD television because most of the time it is viewed only by one person.

Most of TR-LCDs require either a quarter-wave plate (QWP) (Ref. 5) or a patterned in-cell phase retarder ${ }^{6,7}$ in order to obtain a good dark state for the $R$ mode. The major problem associated with the conventional QWP-based broadband circular polarizer is its rather narrow viewing angle. ${ }^{8,9}$ Although forming patterned in-cell phase retarder in the reflective region allows linear polarizers to be employed, the process for fabricating patterned in-cell phase retarder remains a technical challenge.

In this letter, we proposed a single cell gap wide-view TR-LCD using FFS-based LC cell and embedded wire grid polarizer (WGP) which functions as a polarization dependent reflector to avoid the need of any in-cell phase retarder or QWP. An advantage of FFS mode is its inherent wide viewing angle under merely two linear polarizers as compared to twisted nematic or VA modes. ${ }^{10}$ The maximum light efficiency of $T$ and $R$ modes using a positive dielectric anisotropy $(\Delta \varepsilon)$ LC material reaches $\sim 90 \%$ and $\sim 70 \%$, respectively. Moreover, the voltage dependent transmittance $\left(V_{T}\right)$ and reflectance $\left(V_{R}\right)$ curves match well, making it quite suitable for a single gamma curve driving.

Figure 1 shows the schematic device configuration of the proposed transflective LCD. Each pixel of the FFS cell is divided into a $T$ region and a $R$ region, where a WGP (as shown in the inset) is embedded under the reflective electrodes of the indium tin oxide glass substrate as a polarization dependent reflector. Recently, WGPs for visible lights have been fabricated by UV nanoimprinting technology ${ }^{11}$

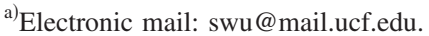

and pixilated WGPs on the thin-film-transistor (TFT) glass substrates have been applied to stereoscopic displays. ${ }^{12}$ For an unpolarized impinging light, the grating structured WGP works like a polarization dependent reflector that reflects the light with its polarization parallel to the metal ribs while transmitting the other component polarized perpendicular to the grids. 13

Figure 2 shows the operation mechanisms for achieving a dark state (voltage off) and a bright state (voltage on) of the proposed TR-LCD. Here, the transmission axis of the top linear polarizer is along the $x$ axis and the boundary LC directors are initially rubbed at $0^{\circ}$ (or $90^{\circ}$ ) with respect to this direction. In the voltage-off state, the linearly polarized ambient light from the top polarizer keeps its polarization after passing through the homogeneous LC cell. With its polarization perpendicular to the wire grids, the light passes through the WGP and is absorbed by the bottom polarizer. For the transmissive part, the backlight passing the bottom polarizer maintains its polarization state throughout the LC layer and is blocked by the crossed top polarizer. Consequently, a common dark state is achieved for both $T$ and $R$ modes.

On the other hand, as the applied voltage exceeds a threshold voltage, the LC directors are reoriented by the electric fields generated from the pixel and common electrodes, as shown in Fig. 1. As depicted in Fig. 2, the stripe pixel electrodes are formed with an angle of $\phi$ with respect to the LC rubbing direction in the $x-y$ plane to gain an optimum

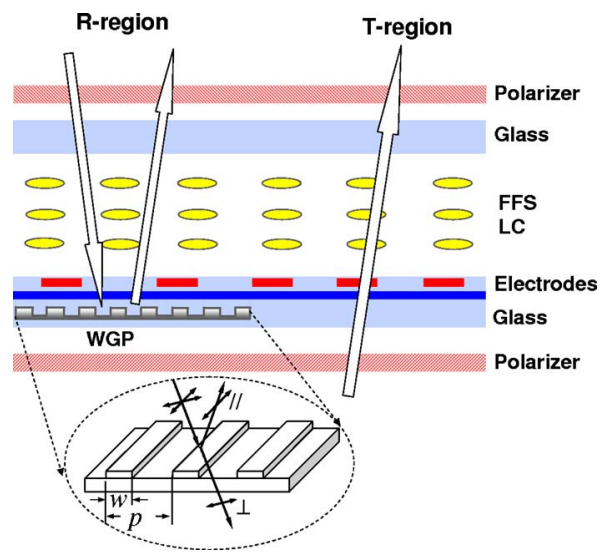

FIG. 1. (Color online) Schematic structure of the single cell gap FFS transflective LCD device using embedded wire grid polarizer as reflector. 


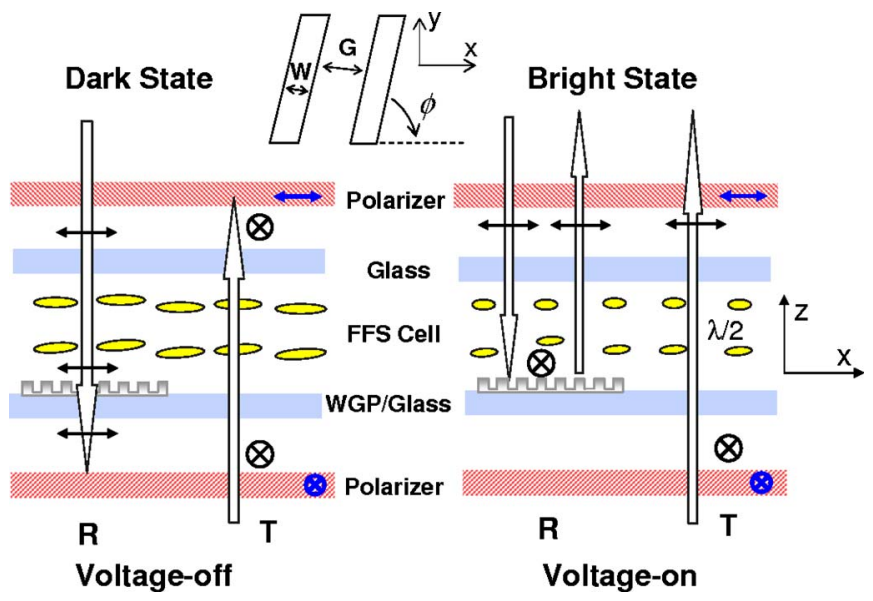

FIG. 2. (Color online) Working principles of the $T$ and $R$ modes at both dark (voltage off) and bright (voltage on) states.

rotation. ${ }^{4}$ Under proper reorientation (about $45^{\circ}$ away from its initial orientation), the LC layer functions like a $\lambda / 2$ plate. In the $R$ part, the polarization axis of the incoming ambient light from the top polarizer is rotated $90^{\circ}$ by the LC cell and becomes parallel to the wire grids, thus, is reflected back to the LC cell by the WGP. It further transverses the $\lambda / 2 \mathrm{LC}$ cell and gets another $90^{\circ}$ rotation and thus, exits the top polarizer. Similarly in the $T$ mode, the backlight from the bottom polarizer is also rotated by the LC cell to transmit the top polarizer. As a result, a common bright state is achieved.

At an intermediate gray level, the effective phase retardation of the LC layer is less than $\lambda / 2$. The incident light from the $R$ part reaching the WGP surface has an elliptical polarization, i.e., it has both parallel and perpendicular components to the wire grids. The parallel component will be reflected back to the cell, while the component perpendicular to the wire grids transmits the WGP (which will be absorbed by the bottom liner polarizer), resulting in an additional loss, as compared to the $T$ mode. In other words, the reflectance at an intermediate gray level is always lower than that of the $T$ part. Moreover, the $R$ mode exhibits a higher thresholdlike voltage than the $T$ mode. The explanation is as follows. As the voltage is slightly higher than the threshold of the $T$ mode, the transmittance starts to increase. However, for the $R$ mode, the light incident onto WGP surface still has most of its components perpendicular to the wire grids, and only a small parallel portion is reflected. Upon reflection, the reflected light from WGP is optically equivalent to the light coming from backlight (for the $T$ part) that has just transmitted the bottom linear polarizer, except its relative intensity is much weaker. As a result, no noticeable increase in reflectance is observed until a higher voltage is reached. This delayed response makes $R$ mode exhibit a slightly higher threshold voltage which, in turn, separates the $V_{T}$ and $V_{R}$ curves. To solve this problem, we can tune the threshold voltage of the $T$ part a little bit higher to match that of the $R$ part by adjusting the electrode width $W$ and gap $G$ in the $T$ and $R$ regions.

To prove the concept, the $V_{T}$ and $V_{R}$ curves using a $+\Delta \varepsilon$ LC material are calculated using a commercial simulation software 2DIMMOS (from autronic-MELCHERS, Germany). The LC mixture employed in simulations is Merck MLC6686; its physical properties are listed as follows: extraordinary and ordinary refractive indices $n_{e}=1.5574, n_{o}=1.4824$

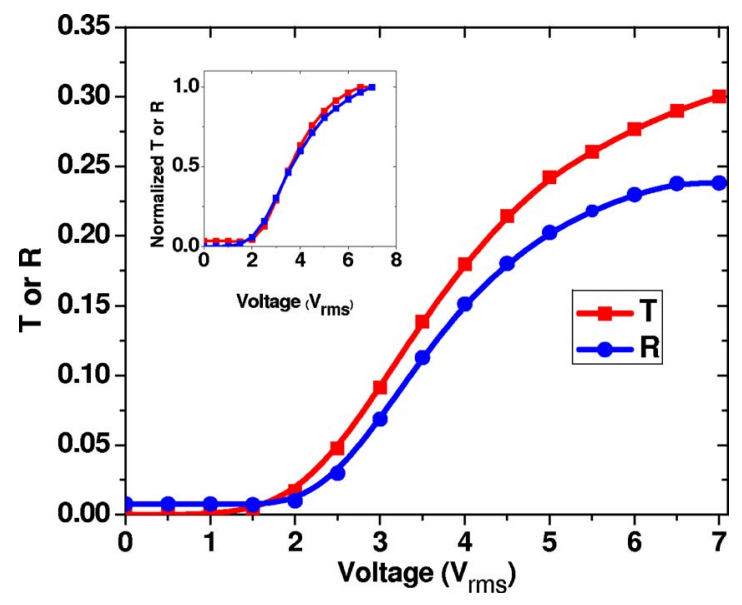

FIG. 3. (Color online) $V_{T}$ and $V_{R}$ curves for the proposed TR-LCD. The inset shows the normalized results.

(at $\lambda=589 \mathrm{~nm}$ ), parallel and perpendicular dielectric constants $\varepsilon_{\|}=14.5, \varepsilon_{\perp}=4.5$, and elastic constants $K_{11}=8.8 \mathrm{pN}$ and $K_{33}=14.6 \mathrm{pN}$. The FFS cell has a cell gap $d=5 \mu \mathrm{m}$ and $2^{\circ}$ surface pretilt angle. The top stripe shaped electrodes is at an angle $\phi \sim 10^{\circ}$ with respect to the LC rubbing direction (making the electric fields $\sim 80^{\circ}$ to the initial LC directors), as Fig. 2 shows. In calculations, the $4 \times 4$ matrix method ${ }^{14,15}$ is used for modeling the $R$ mode and the incident wavelength is $\lambda=550 \mathrm{~nm}$. To find the intrinsic contrast ratio, surface reflections of all the optical components are ignored. The grids of the WGP are along the $y$ axis. The WGP is modeled as a uniaxial layer by the effective media theory ${ }^{13}$ with its ordinary and extraordinary refractive indices as

$$
\begin{aligned}
& n_{o}^{2}=n_{1}^{2} \frac{w}{p}+n_{2}^{2}\left(1-\frac{w}{p}\right), \\
& \frac{1}{n_{e}^{2}}=\frac{1}{n_{1}^{2}} \frac{w}{p}+\frac{1}{n_{2}^{2}}\left(1-\frac{w}{p}\right) .
\end{aligned}
$$

For the present example, the $w / p$ ratio defined as the metal rib width $w$ over the grating pitch $p$, as shown in Fig. 1, is set at 0.2 , the metal for wire grids is made of aluminum with refractive index $n_{1}=0.95+6.69 \times i$ at $\lambda=550 \mathrm{~nm}$, and the gap is filled with dielectric material $\mathrm{SiO}_{2}$ with refractive index $n_{2}=1.48$ for the real TFT processing condition. Accordingly, the effective ordinary and extraordinary refractive indices of the WGP are $n_{o}=0.4724+2.6910 \times i$ and $n_{e}=1.6643$ $+0.0028 \times i$, and $n_{e}$ is along the $x$ axis. As discussed above, the additional loss from the WGP will result in an increased thresholdlike voltage $\left(V_{\mathrm{th}}\right)$ for the reflective mode. In order to compensate this, the electrode width and gap in the $T$ and $R$ regions are set to be different. For example, the electrode width $W_{T}$ and electrode gap $G_{T}$ in the $T$ region are both chosen to be $3 \mu \mathrm{m}$, but in the $R$ region $W_{R}=3 \mu \mathrm{m}$ and $G_{R}$ $=5 \mu \mathrm{m}$. A finer electrode pattern usually leads to higher threshold and on-state voltages in a FFS cell.

As plotted in Fig. 3, the peak transmittance is $\sim 30 \%$ and peak reflectance is $\sim 23.8 \%$, while the maximum transmittance from two parallel linear polarizers is $\sim 33.7 \%$. Thus, the effective transmittance and reflectance reaches $\sim 90 \%$ and $70.6 \%$, respectively. This reduced reflectance originates from two reasons: (1) the nonideal rotation of LC directors in the cell region, such as the LC tilts induced by the fringe fields (LC directors with a $+\Delta \varepsilon$ will be tilted up by the ver- 

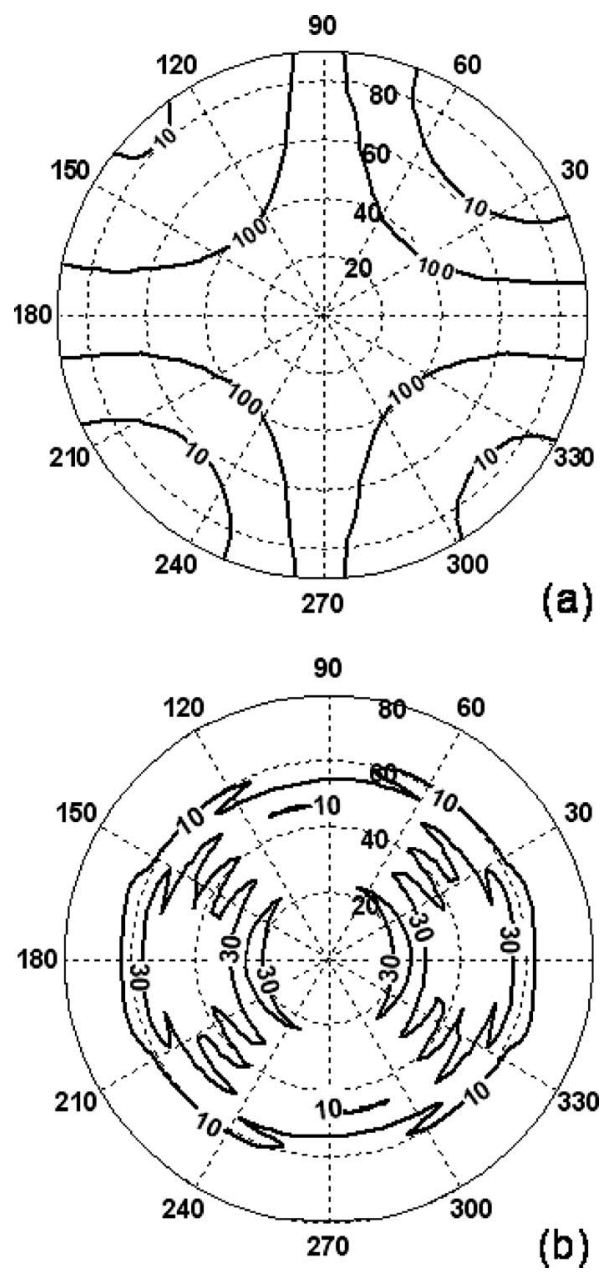

FIG. 4. Isocontrast plots for (a) $T$ mode and (b) $R$ mode of the proposed TR-LCD.

tical fields, resulting in a phase loss) and (2) the imperfect reflection from the WGP, which results from the small imaginary part of the ordinary refractive index of the WGP (only $\sim 2.69$, as compared to 6.69 of pure aluminum). Here, the small light leakage of the incident light below threshold voltage in the $V_{R}$ curve originates from the mismatch between the real part of WGP's extraordinary refractive index $(\sim 1.66)$ and that of the LC material $(\sim 1.55)$, and the internal reflections from other layers. Although the peak reflectance is reduced, the normalized $V_{T}$ and $V_{R}$ curves still overlap quite well, as shown in the inset of Fig. 3, which indicates a single gray scale driving circuit can be employed for this display.

Another feature of this display is its wide viewing angle without using any phase compensation films. From Fig. 4(a), the $T$ mode shows an inherently wide viewing angle with its $C_{R}>10: 1$ viewing cone over $60^{\circ}$ at all directions. For the $R$ mode, although a small light leakage at $V=0$ exists, as Fig. 3 shows, its viewing angle is also quite wide, as observed from Fig. 4(b). The $C_{R}>10: 1$ contour spans over the $50^{\circ}$ viewing cone without using any QWP or in-cell phase retarder. This viewing angle is adequate for mobile display applications.

The reasonably wide viewing angle in the $R$ mode originates from two factors: (1) the LC directors in the FFS cell are aligned perpendicular to the absorption axis of the top linear polarizer (which also accounts for the wide viewing angle for the $T$ mode) and (2) the additional absorption of oblique incident lights on the WGP surface. For example, let us consider an unpolarized light incident on the panel at $\sim 50^{\circ}$ polar angle and $\sim 45^{\circ}$ azimuthal angle. This light will have polarization components both parallel and perpendicular to the wire grids when it reaches the WGP surface and only part of them is reflected, making light leakage further suppressed. Meanwhile, as Fig. 4(b) shows, the contrast ratios along the transmission axis of the top linear polarizer (where azimuthal angle $=0^{\circ}$ or $180^{\circ}$ ) are the largest as compared to other directions, such as the $90^{\circ}$ direction. This is because for an off-axis incidence at this direction, the incident light passing through the top linear polarizer only has a light component whose polarization is perpendicular to the wire grids; the other component is absorbed by the top polarizer. As a result, it transmits through the WGP and is absorbed by the bottom linear polarizer. Conversely, for the incident light at other directions, the light (after passing the top linear polarizer) still has both components that are parallel and perpendicular to the wire grids, and only part of them can be suppressed by the WGP.

In conclusion, we have demonstrated a transflective FFS LCD employing a pixilated WGP as reflector. This device does not require any in-cell phase retarder or quarter-wave plate and has a single cell gap configuration. Both $T$ and $R$ modes exhibit a reasonably high optical efficiency and wide viewing angle. In addition, the $T$ and $R$ modes could share a single grayscale gamma curve. With the progress of nanoimprinting of visible wire grid polarizers, we believe this design has a great application potential for future high performance transflective LCDs.

The authors are indebted to the financial support from Chi-Mei Optoelectronics, Taiwan.

${ }^{1}$ For a review, see X. Zhu, Z. Ge, T. X. Wu, and S. T. Wu, J. Disp. Technol. 1, 15 (2005).

${ }^{2}$ A. Takeda, S. Kataoka, T. Sasaki, H. Chida, H. Tsuda, K. Ohmuro, Y. Koike, T. Sasabayashi, and K. Okamoto, SID Int. Symp. Digest Tech. Papers 29, 1077 (1998).

${ }^{3}$ J. O. Kwag, K. C. Shin, J. S. Kim, S. G. Kim, and S. S. Kim, SID Int. Symp. Digest Tech. Papers 31, 256 (2000).

${ }^{4}$ S. H. Lee, S. L. Lee, and H. Y. Kim, Appl. Phys. Lett. 73, 2881 (1998).

${ }^{5}$ S. T. Wu and C. S. Wu, Appl. Phys. Lett. 68, 1455 (1996).

${ }^{6}$ J. H. Song, Y. J. Lim, M. H. Lee, S. T. Shin, and S. H. Lee, Appl. Phys. Lett. 87, 011108 (2005).

${ }^{7}$ J. B. Park, H. Y. Kim, Y. H. Jeong, S. Y. Kim, and Y. J. Lim, Jpn. J. Appl. Phys., Part 1 44, 7524 (2005).

${ }^{8}$ S. Pancharatnam, Proc. Indian Acad. Sci., Sect. A 41, 130 (1955).

${ }^{9}$ Q. Hong, T. X. Wu, R. Lu, and S. T. Wu, Opt. Express 13, 10777 (2005).

${ }^{10}$ Z. Ge, X. Zhu, and S. T. Wu, J. Disp. Technol. 2, 102 (2006).

${ }^{11}$ J. Wang, L. Chen, X. Liu, P. Sciortino, F. Liu, F. Walters, and X. Deng, Appl. Phys. Lett. 89, 141105 (2006).

${ }^{12}$ J. H. Oh, D. H. Kang, W. H. Park, H. J. Kim, S. M. Hong, K. H. Hur, J. Jang, S. J. Lee, M. J. Kim, S. K. Kim, K. H. Park, E. Gardner, J. Hansen, M. Yost, and D. Hansen, SID Int. Symp. Digest Tech. Papers 38, 1164 (2007).

${ }^{13}$ X. J. Yu and H. S. Kwok, J. Appl. Phys. 93, 4407 (2003).

${ }^{14}$ D. W. Berreman, J. Opt. Soc. Am. 62, 502 (1972).

${ }^{15}$ Z. Ge, X. Zhu, T. X. Wu, and S. T. Wu, J. Opt. Soc. Am. A 22, 966 (2005). 